Steven R. Pride · James G. Berryman

\title{
Goddard rattler-jamming mechanism for quantifying pressure dependence of elastic moduli of grain packs
}

Received: 18 December 2008 / Revised: 9 January 2009 / Published online: 4 April 2009

(C) The Author(s) 2009. This article is published with open access at Springerlink.com

\begin{abstract}
An analysis is presented to show how it is possible for unconsolidated granular packings to obey overall non-Hertzian pressure dependence due to the imperfect and random spatial arrangements of the grains in these packs. With imperfect arrangement, some gaps that remain between grains can be closed by strains applied to the grain packing. As these gaps are closed, former rattler grains become jammed and new stressbearing contacts are created that increase the elastic stiffness of the packing. By allowing for such a mechanism, detailed analytical expressions are obtained for increases in bulk modulus of a random packing of grains with increasing stress and strain. Only isotropic stress and strain are considered in this analysis. The model is shown to give a favorable fit to laboratory data on variations in bulk modulus due to variations in applied pressure for bead packs.
\end{abstract}

\section{Introduction}

Unconsolidated sediments form an important class of earth materials because their large storage capacity makes them ideal fluid reservoirs. They are therefore important as repositories for $\mathrm{CO}_{2}$, as oil reservoirs, and as drinking water aquifers. Seismic monitoring of changes in these reservoirs as fluids are pumped into or out of them is of importance to society. Furthermore, unconsolidated ocean-bottom sediments are important for long-range sound propagation in the ocean, and, therefore, in marine acoustic studies of the seafloor.

One key to understanding these applications is knowledge of how moduli of the elastic framework change as either the fluid pressure in the pores changes or the confining pressure acting on the grain pack (e.g., the overburden pressure) changes. It is well established experimentally (e.g., [1,2]) that the elastic moduli of grain packs are independent of the grain sizes. If all the grains in a pack are uniformly reduced or expanded in a self-similar fashion, the elastic moduli will not change. Such scale-invariance means that incremental changes in the elastic moduli will occur only if the effective-stress increment $\delta P=\delta P_{c}-\alpha_{\phi} \delta P_{f}$ is non-zero $[3,4]$, where $P_{c}$ is the confining pressure acting on the pack, $P_{f}$ is the fluid pressure, and $\alpha_{\phi}$ is the porosity effective-stress coefficient, which is unity if all the grains are made of the same material-as will be assumed

S. R. Pride · J. G. Berryman ( $\varangle)$

Earth Sciences Division, Lawrence Berkeley National Laboratory,

One Cyclotron Road MS 90R1116, Berkeley, CA 94720, USA

E-mail: jgberryman@lbl.gov

Tel.: +510-486-5349

Fax: +510-486-5686

S. R. Pride

E-mail: srpride@lbl.gov

Tel.: +510-495-2823

Fax: +510-486-5686 
here. Thus, the elastic moduli of grain packs under isotropic compression only depend on the effective stress combination $P=P_{c}-P_{f}$.

Unconsolidated sediments have distinct properties compared to cemented sandstones. Perhaps chief among them is that, when stress is applied to random grain packings, there develops a surprisingly complicated internal stress distribution that can be described either as "force chains" or "stress bridges" (e.g., [5-7]). Such stress distributions can be visualized in photoelastic experiments using birefringent beads (either plastic or glass). When the beads become stressed, light passing through them becomes polarized so images can be made showing which beads in the packing support the greatest stress. In recent experiments of Majmudar and Behringer [8], it is directly observed that even in the case of isotropic compression, the internal distribution of stress is far from uniform and may be considered randomly oriented force chains, isotropically distributed in all directions.

For random loose packing of grains, as many as $10 \%$ of the grains may be experiencing no stress whatsoever at low strain levels [9]. Such grains are called "rattlers," because there remains a certain degree of rattling room between themselves and their immediate neighbors. As strain is applied to the packing, the grain-to-grain gap size diminishes until individual rattlers finally become "jammed" and these new additions to the backbone begin to accumulate stress. Modeling how the rattlers become jammed with increasing strain is important for our understanding of the pressure dependence in the elastic properties of grain packs.

In the present article, we first briefly review the well-know discord between the Hertzian pressure dependence of the elastic moduli $\left(P^{1 / 3}\right)$ and the pressure dependence often measured in laboratory experiments (closer to $P^{1 / 2}$ ). We then follow Goddard [10], and present two mechanisms for how rattlers become jammed as a function of applied strain and show how to incorporate such mechanisms of gap closure into an analytical model for the elastic moduli.

\section{Hertzian contact mechanics versus measured pressure dependence of actual grain packs}

Walton [11] derives compact analytical expressions for the drained moduli of grain packs based on Hertzian contact mechanics [12] between spheres. The theory assumes that each sphere in the pack has a center that is displacing according to the macroscopic strain field applied to the entire packing. Furthermore, the coordination number of stress-contributing contacts is not assumed to evolve with the changing applied strain. For isotropic applied strain $\epsilon$ (defined positive in compression and such that $3 \epsilon$ corresponds to overall volumetric strain), the Walton theory [11] predicts that the relation between the overall pressure in the pack $P$ and strain $\epsilon$ is

$$
P=\frac{\left(1-\phi_{0}\right) n_{w}}{3 \pi^{2} B_{w}} \epsilon^{3 / 2},
$$

where $n_{w}$ is the coordination number (average number of stress-bearing contacts per sphere), $\phi_{0}$ is the porosity of the random packing at $P=0$, and $B_{w}$ is a compliance parameter, defined by

$$
B_{w}=\frac{1}{4 \pi}\left(\frac{1}{G_{s}}+\frac{1}{K_{s}+G_{s} / 3}\right)
$$

where $G_{s}$ and $K_{s}$ are the shear and bulk moduli, respectively, of the grain material. Equation (1) yields a bulk modulus $K$ given by

$$
K \equiv \frac{\mathrm{d} P}{3 \mathrm{~d} \epsilon}=\frac{1}{2}\left[\frac{\left(1-\phi_{0}\right) n_{w}}{3 \pi^{2} B_{w}}\right]^{2 / 3} P^{1 / 3} .
$$

The factor of three in the definition of the bulk modulus follows because of Walton defining $\epsilon$ to be three times smaller (in 3D) than the total volumetric strain. Walton's theory [11] thus predicts a $P^{1 / 3}$ (or "Hertzian") pressure dependence for the bulk modulus of the drained frame.

The shear modulus in the Walton [11] model is given as

$$
G=R K,
$$

where the parameter $R$ takes on a value somewhere in the range

$$
\frac{3}{5} \leq R \leq \frac{18}{5}\left(\frac{K_{s}+G_{s}}{3 K_{s}+2 G_{s}}\right)
$$


The lower limit corresponds to grains so smooth that frictionless tangential slip always occurs, which prevents shear force from being transmitted at the contact. Similarly, the upper limit corresponds to grains so rough that no slip occurs, resulting in the maximum transmitted shear at a contact.

Acoustic data collected on unconsolidated sand and glass bead packs as presented in numerous investigations [1,2,13-17] demonstrate that over the approximate stress range of $10^{-3}$ to $10 \mathrm{MPa}$ (corresponding to sediment overburden depths ranging from $10^{-1}$ to $10^{3} \mathrm{~m}$ ), elastic moduli of the dry frame vary more rapidly than the Hertzian prediction of $P^{1 / 3}$. At lower levels of applied pressure $(<10 \mathrm{MPa})$, power-law pressuredependence is generally observed to have an exponent larger than the Hertzian $1 / 3$. However, at higher ranges of pressure, the pressure dependence in many of these studies does tend to transition to the idealized Hertzian result.

Hardin and Richart [1] and Domenico [13] obtain an approximate $P^{1 / 2}$ pressure-dependence of the moduli up to their maximum pressures which were not high enough to see a transition to Hertzian dependence. Makse et al. [14] also observe a $P^{1 / 2}$ pressure dependence; however, their experiments are confined to the extremely high pressure range of 50-140 MPa. Typically, glass beads begin to break at pressures in this range, and therefore a different mechanism - possibly right at the grain-to-grain contact interfaces-may be necessary to explain their observed pressure dependence.

Focusing only on studies of glass bead samples under isotropic compression, Kuwano and Jardine [15] measure a steeper pressure dependence of roughly $P^{0.6}$ over the relatively low pressure range from 0.05 to $0.4 \mathrm{MPa}$. Murphy [2] also measures a pressure dependence of $P^{0.6}$ over $0.2-2 \mathrm{MPa}$ of applied pressure before the data trend over to the Hertzian dependence near $P=20 \mathrm{MPa}$. Zimmer et al. [17] measure an even steeper pressure dependence of roughly $P^{0.8}$ on their glass-bead samples at pressures from 0.1 to $0.4 \mathrm{MPa}$ before the data trend off to the Hertzian dependence when $P>1 \mathrm{MPa}$. However, Jia and Mills [16] measure a pressure dependence for their glass-bead packs that is very close to the Hertzian prediction over the pressure range from 0.07 to $0.8 \mathrm{MPa}$.

Clearly, one single universal pressure exponent does not emerge from all of these experimental studies, even when only glass beads under isotropic compression are compared. The main differences among these various studies probably lies in details of how the bead samples were prepared; i.e., in the nature of the disordered state where each grain pack starts at zero stress and strain. In the present model, the nature of the randomness in the pack at zero stress will emerge as a key factor to explain various pressure dependencies that have been observed.

\section{Goddard mechanism for creating force-bearing contacts}

To account for the observed non-Hertzian pressure dependence of the moduli at lower pressures, we follow Goddard [10] and allow for new force-bearing contacts to be created as strain is applied to the grain packs. Although Goddard's ideas [10] have certainly received some attention $[9,14,17-21]$ in the granular packing literature, the present authors feel more attention to the details is appropriate, and this is the main contribution that is being made here. Goddard [10] was motivated to demonstrate that the elastic moduli increase with pressure as $P^{1 / 2}$, instead of $P^{1 / 3}$ (which is again the result due to Hertz). Our goal is to explain the range of experimentally observed exponents on the pressure power law which will require the introduction of several new ideas and assumptions not made specifically by Goddard [10]. In the following discussion, we point out when Goddard's ideas [10] are being used.

We imagine starting with a diffuse collection of grains occupying random positions in a region bounded by a surface that can either be expanded or shrunken. We compress the region isotropically until grain contacts first percolate across the system. Starting from this point, which corresponds to a random loose pack in a state of zero strain, any additional compression of the region will require work to be performed and stress to accumulate.

When the grain-to-grain contacts first percolate, additional compression will create force at contacts on the backbone only if the grains along this incipient force chain have no rattling room left to them; i.e., the grains along the backbone must all be jammed in order for force to build. If there is a gap between a grain on the backbone and a lateral nearest neighbor, compression in the direction of the particle chain can result in a local grain rotation until the gap is closed and contact with the stabilizing lateral neighbor is made. This idea is the fundamental one presented by Goddard [10]. Alternatively, the gaps may close, not by grain rotation, but by simple linear strain accumulation. Both mechanisms may be present simultaneously, and both will be considered here. 


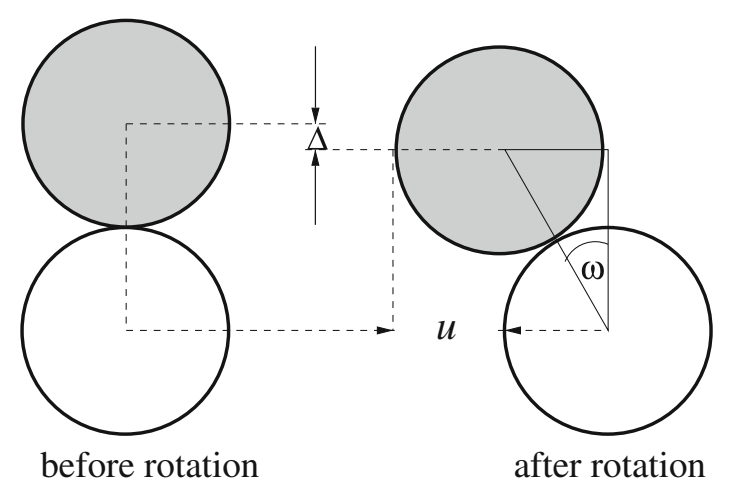

Fig. 1 Illustrating Goddard's [10] concept that a rattler grain (shown in grey) may rotate a lateral distance $u$ in order to accommodate strain $\Delta / D$ (where $D$ is grain diameter) imposed in the direction of the initial line segment connecting adjacent grain centers unless it is prevented from doing so by the presence of a sphere (not shown) in the lateral direction. Note that the final configuration could have also been achieved through particle-particle slippage, assuming the friction between the grains is very small

Although we use the term "grain rotation" to be consistent with Goddard [10], it should nevertheless be noted that when slippage is possible between grains (due to low grain-to-grain friction, as is allowed in the Walton model), the final result of the particle movement can be indistinguishable from a grain rotation, as noted in Fig. 1.

To begin quantifying these notions, we define $\frac{1}{2} n N$ as the number of force bearing contacts throughout the pack of $N$ grains at any given instant in strain history. Note that the coordination number $n$ defined in this way necessarily satisfies $0 \leq n \leq 12$ in 3D and represents the average number of force-bearing contacts on each sphere in the pack. A first goal is to determine the increment in the number of force-bearing contacts $\frac{1}{2} N \mathrm{~d} n$, when the isotropic strain acting on the pack (defined as positive in compression) is increased from $\epsilon$ to $\epsilon+d \epsilon$. At $\epsilon=0$, we assume that there is a total of $\frac{1}{2} n_{0} N$ contacts along a percolating backbone capable of bearing force. There are also a total of $N_{r}$ unjammed (or "rattler") grains throughout the pack. We will assume that after all $N_{r}$ of the rattlers have made contact with their lateral neighbors (and thus become jammed), the number of force-bearing contacts is $\frac{1}{2} n_{\infty} N$, and it does not continue to increase thereafter with increasing strain.

On average, $\frac{1}{2}\left(n_{\infty}-n_{0}\right) N / N_{r}$ force-bearing contacts are created each time a rattler makes contact with a neighbor. At any point in strain history, the number of rattlers that have already made contact with a neighbor is then the total number of force-bearing contacts created by the jamming of rattlers, $\frac{1}{2}\left(n-n_{0}\right) N$, divided by the number of force-bearing contacts created each time a gap is closed, $\frac{1}{2}\left(n_{\infty}-n_{0}\right) N / N_{r}$. So the probability that a given rattler has not yet made contact with a neighbor is $1-\left(n-n_{0}\right) /\left(n_{\infty}-n_{0}\right)$. We then find that

$$
N \mathrm{~d} n=N_{r}\left(1-\frac{n-n_{0}}{n_{\infty}-n_{0}}\right) p(u) \mathrm{d} u \frac{\left(n_{\infty}-n_{0}\right) N}{N_{r}},
$$

or, equivalently,

$$
\frac{\mathrm{d}\left(n-n_{0}\right) /\left(n_{\infty}-n_{0}\right)}{1-\left(n-n_{0}\right) /\left(n_{\infty}-n_{0}\right)}=p(u) \mathrm{d} u .
$$

Here, $p(u) d u$ is the probability that a neighbor was positioned within a distance $u+\mathrm{d} u$ of a rattler grain, where $u$ is the amount of displacement undergone by a rattler when the strain level is $\epsilon$.

The probability density $p(u)$ of gaps to nearest neighbors is unfortunately not usually measured in numerical studies of random grain packs. We therefore assume for the sake of argument that it is a power law given by

$$
p(u)=\frac{m u^{m-1}}{h^{m}} ; \quad 0<u<h,
$$

where the exponent $m$ must be positive and where $h$ is the initial maximum gap size to a nearest neighbor. The length $h$ must necessarily be a small fraction of the grain diameter $D$ if the initial random state has percolating 
contacts at $\epsilon=0$. The value of the power-law exponent $m$ becomes an adjustable parameter that can be used to fit the observed pressure dependence of the moduli. Physically reasonable values for the parameter $m$ in (8) lie in the range $0^{+}<m \leq 1$. Values of $m$ greater than one correspond to larger gaps being more probable than smaller gaps which we assume is never the situation in naturally prepared grain packs.

Integrating Eq. (7) using the probability density of Eq. (8) yields

$$
\ln \left(1-\frac{n-n_{0}}{n_{\infty}-n_{0}}\right)=\ln \left(\frac{n_{\infty}-n}{n_{\infty}-n_{0}}\right)=-\left(\frac{u}{h}\right)^{m} .
$$

To proceed, we need models relating the displacement $u$ to the applied strain $\epsilon$.

We consider first the rotation mechanism proposed by Goddard [10] and depicted in Fig. 1. If $D$ is the grain diameter (assumed the same for all grains), the local strain $\epsilon_{\ell}=\Delta / D$ along the line segment initially joining the two sphere centers can be accommodated by a force-free rotation $\omega$ if there are no lateral neighbors to inhibit the rotation, and/or slippage. As the figure clearly shows, $\cos \omega=(D-\Delta) / D=1-\epsilon_{\ell}$. For small rotations this yields $\epsilon_{\ell}=\omega^{2} / 2$ or $\omega=\sqrt{2 \epsilon_{\ell}}$. The lateral displacement $u$ created by such rotation is $u=D \sin \omega \simeq D \sqrt{2 \epsilon_{\ell}}$. It will be assumed here that the line segments connecting neighboring sphere centers are isotropically oriented throughout the pack so that the local strain $\epsilon_{\ell}$ being accommodated by the rotation is the overall isotropic strain being applied to the system as a whole; i.e., $\epsilon_{\ell}=\epsilon$.

Alternatively, and more simply, one may assume that the gaps are closed by linear strain accumulation $u=D \epsilon$. Goddard [10] stated that for this mechanism to explain the observed pressure dependence of the elastic moduli, special distribution functions must be assumed for the gap sizes. He thus dismissed the closing of the gaps by linear strain accumulation out of hand. We will come to somewhat different conclusions on this issue.

Both mechanisms of gap closure can be expressed in the unified expression

$$
u=D(\chi \epsilon)^{1 / \chi}
$$

where $\chi$ is the closure index and takes the value $\chi=1$ for linear strain accumulation, and $\chi=2$ for grain rotation. Substituting $u=D(\chi \epsilon)^{1 / \chi}$ into Eq. (9), we find the desired expression for how the coordination number $n(\epsilon)$ of force-bearing contacts varies with isotropic strain:

$$
n(\epsilon)=n_{\infty}-\left(n_{\infty}-n_{0}\right) \mathrm{e}^{-\alpha_{m} \epsilon^{m / x}}
$$

where the parameter $\alpha_{m}$ is defined by

$$
\alpha_{m}=\left(\frac{D \chi^{1 / \chi}}{h}\right)^{m}
$$

As required, this expression for $n(\epsilon)$ reduces to $n_{0}$ at zero strain and $n_{\infty}$ at very large strain. A transition strain value $\epsilon_{t}$ can be identified by

$$
\epsilon_{t}=\alpha_{m}^{-\chi / m}
$$

thereby separating low-strain power-law increases in coordination number

$$
n \sim n_{0}+\left(n_{\infty}-n_{0}\right)\left(\frac{\epsilon}{\epsilon_{t}}\right)^{m / \chi}, \text { for } \frac{\epsilon}{\epsilon_{t}} \ll 1,
$$

from the high-strain limit

$$
n \sim n_{\infty}, \quad \text { for } \frac{\epsilon}{\epsilon_{t}} \gg 1
$$

where the coordination number remains constant.

In the next section, we need to know how many new force-bearing contacts are created in each strain increment, which is

$$
\frac{\mathrm{d} n}{\mathrm{~d} \epsilon}=\frac{\left(n_{\infty}-n_{0}\right) m \alpha_{m}}{\chi} \epsilon^{m / \chi-1} e^{-\alpha_{m} \epsilon^{m / \chi}} .
$$

Note that so long as $m / \chi<1$, the greatest increase in force-bearing contacts occurs at small strain $\epsilon=0^{+}$, and then progressively decreases as $\epsilon$ increases. In the following discussion, all explicit dependence on $m$ and $\chi$ will appear in the ratio $m / \chi$. The only term that depends on the separate values of both $m$ and $\chi$ is the parameter $\alpha_{m}$ defined in Eq. (12). 


\section{Stress and strain dependence of elastic moduli}

The theory of Walton [11] assumes that all force-bearing contacts are already in place and start accumulating force from $\epsilon=0^{+}$. We now show how to generalize the Walton [11] model to allow for force-bearing contacts that arrive at various points along the strain history.

We begin by estimating the amount of Hertzian contact force that accumulates with strain at a contact between jammed spheres. Under the assumption that the local strain is given by the overall applied strain, the Walton [11] result for the Hertzian force at contact $c$ can be written as

$$
\mathbf{F}^{c}=\frac{D^{2}}{3 \pi B_{w}}(\epsilon-s)^{3 / 2} \mathbf{I}^{c},
$$

where $B_{w}$ is Walton's compliance parameter given herein by Eq. (2), $D$ is the grain diameter, $s$ is the isotropic strain level at which contact $c$ first begins to accumulate force, and $\mathbf{I}^{c}$ is the unit vector along the line segment connecting the two sphere centers. Upon putting $s=0$, Eq. (17) above is just Eq. (3.7) from [11]. The assumption that local strain is uniform throughout the pack is of course not formally correct once stress starts to accumulate in patches. The main error incurred in making this assumption is that the distribution function for the local contact forces in the present model will not be correct, as will be discussed later.

For a mono-disperse packing of spheres, Walton [11] shows that the volume-averaged stress tensor $\left\langle\sigma_{i j}\right\rangle$ acting throughout a pack of volume $V$ is exactly related to the contact forces as

$$
\left\langle\sigma_{i j}\right\rangle=-\frac{D}{2 V} \sum_{c}\left(I_{i}^{c} F_{j}^{c}+I_{j}^{c} F_{i}^{c}\right)
$$

where the sum is over all contacts in the volume. Putting Eq. (17) into Eq. (18), and replacing the sum over contacts by a number density integral over contacts, yields

$$
\left\langle\sigma_{i j}\right\rangle=-\frac{N D^{3}}{12 \pi B_{w} V}\left[n_{0} \epsilon^{3 / 2}\left\langle I_{i} I_{j}+I_{j} I_{i}\right\rangle_{o}+\int_{0}^{\epsilon} \mathrm{d} s(\epsilon-s)^{3 / 2} \frac{\mathrm{d} n}{\mathrm{~d} s}\left\langle I_{i} I_{j}+I_{j} I_{i}\right\rangle_{s}\right] .
$$

The brackets \langle\rangle$_{s}$ indicate an average over all contacts created in the strain interval $s \rightarrow s+\mathrm{d} s$, while $\frac{1}{2} N(\mathrm{~d} n / \mathrm{d} s) \mathrm{d} s$ is the number of new force-bearing contacts created in the interval $s \rightarrow s+\mathrm{d} s$ with $\mathrm{d} n / \mathrm{d} s$ given by Eq. (16).

For randomly oriented unit vectors $\mathbf{I}$, one has $\left\langle I_{x}^{2}\right\rangle_{s}=\left\langle I_{y}^{2}\right\rangle_{s}=\left\langle I_{z}^{2}\right\rangle_{s}=\langle\mathbf{I} \cdot \mathbf{I}\rangle_{s} / 3=1 / 3$, while $\left\langle I_{x} I_{y}\right\rangle_{s}=0$, etc., which leads to

$$
\left\langle I_{i} I_{j}+I_{j} I_{i}\right\rangle_{s}=\frac{2}{3} \delta_{i j}
$$

Furthermore, at zero strain, the volume occupied by $N$ grains in volume $V$ is $N \pi D^{3} / 6$, which allows the zero strain porosity $\phi_{0}$ of the packing to be defined by

$$
1-\phi_{0}=\frac{N \pi D^{3}}{6 V}
$$

Introducing the overall pressure $P$ as $\left\langle\sigma_{i j}\right\rangle=-P \delta_{i j}$ and using Eq. (16) for $\mathrm{d} n / \mathrm{d} s$ yields

$$
P=\frac{\left(1-\phi_{0}\right)}{3 \pi^{2} B_{w}}\left[n_{0} \epsilon^{3 / 2}+\left(n_{\infty}-n_{0}\right) \frac{m \alpha_{m}}{\chi} J_{\epsilon}\right],
$$

where the strain integral $J_{\epsilon}$ is defined by

$$
J_{\epsilon}=\int_{0}^{\epsilon}(\epsilon-s)^{3 / 2} s^{m / \chi-1} \mathrm{e}^{-\alpha_{m} s^{m / \chi}} \mathrm{d} s .
$$


Upon making the substitution of variables $x=\sqrt{s}, \mathrm{~d} x=\mathrm{d} s /(2 \sqrt{s})$ and $\xi \equiv \sqrt{\epsilon}$, the strain integral $J_{\epsilon}$ becomes

$$
\begin{aligned}
J_{\epsilon} & =2 \int_{0}^{\xi}\left(\xi^{2}-x^{2}\right)^{3 / 2} x^{2(m / x-1)} \mathrm{e}^{-\alpha_{m} x^{2 m / x}} \mathrm{~d} x \\
& =2 \sum_{k=0}^{\infty} \frac{\left(-\alpha_{m}\right)^{k}}{k !} \int_{0}^{\xi}\left(\xi^{2}-x^{2}\right)^{3 / 2} x^{2(k+1) m / \chi-1} \mathrm{~d} x,
\end{aligned}
$$

which is a tabulated form (e.g., [22]) giving

$$
J_{\epsilon}=\sum_{k=0}^{\infty} \frac{\left(-\alpha_{m}\right)^{k}}{k !} B\left(\frac{(k+1) m}{\chi}, \frac{5}{2}\right) \epsilon^{(1+k) m / \chi+3 / 2} .
$$

The convention $0 !=1$ is being employed, while $B(a, b)$ is the so-called beta function which is related to the gamma function as $B(a, b)=\Gamma(a) \Gamma(b) / \Gamma(a+b)$.

The resulting relationship between pressure and strain is therefore

$$
P=\frac{\left(1-\phi_{0}\right)}{3 \pi^{2} B_{w}}\left\{n_{0} \epsilon^{3 / 2}+\left(n_{\infty}-n_{0}\right) \frac{m \alpha_{m}}{\chi} B\left(\frac{m}{\chi}, \frac{5}{2}\right) \epsilon^{3 / 2+m / \chi}\left[1+\sum_{k=1}^{\infty} \frac{\left(-\alpha_{m}\right)^{k}}{k !} \beta_{k} \epsilon^{k m / \chi}\right]\right\},
$$

where

$$
\beta_{k}=\frac{B((k+1) m / \chi, 5 / 2)}{B(m / \chi, 5 / 2)} .
$$

The bulk modulus $K \equiv(\mathrm{d} P / \mathrm{d} \epsilon) / 3$ is finally given by

$$
\begin{aligned}
K= & \frac{(1-\phi) \kappa_{m} \alpha_{m} n_{\infty}}{6 \pi^{2} B_{w}}\left(1-\frac{n_{0}}{n_{\infty}}\right) \epsilon^{1 / 2+m / \chi} \\
& \times\left[1+\frac{n_{0} / n_{\infty}}{\left(1-n_{0} / n_{\infty}\right)} \frac{\epsilon^{-m / \chi}}{\kappa_{m} \alpha_{m}}+\sum_{k=1}^{\infty}\left(1+\frac{2 k m / \chi}{3+2 m / \chi}\right) \frac{\left(-\alpha_{m}\right)^{k}}{k !} \beta_{k} \epsilon^{k m / \chi}\right],
\end{aligned}
$$

where

$$
\kappa_{m}=\frac{(3+2 m / \chi)}{3} \frac{m}{\chi} B\left(\frac{m}{\chi}, \frac{5}{2}\right) .
$$

Applying the ratio test [23] to the series in both Eqs. (27) and (29), we find they are convergent since $\lim _{k \rightarrow \infty} \alpha_{m} \epsilon^{m / \chi} / k<1$. In numerical practice, however, when working with a finite number $k_{\max }$ of terms in the series, we find that the results of the summation are valid only if $\alpha_{m} \epsilon^{m / \chi} / k_{\max }<1$. With both $\alpha_{m}$ and $\epsilon$ sufficiently large, and $k_{\max }$ sufficiently small, convergence problems may be encountered; however, for most conditions experimentally explored, the series produces well-behaved results using just a few terms in this expansion. Phenomenological approximations that avoid having to work directly with the series in Eqs. (27) and (29) will be provided later in this section.

As a consistency check on the above expressions, we investigate the limit as the exponent $m \rightarrow 0$. From Eq. (8) for the probability distribution of gap sizes, this limit corresponds to lateral gaps being concentrated at $u=0$, which means that starting at zero strain, all force-bearing contacts are in place. We should therefore expect to recover the Walton [11] results in this limit. To check satisfaction of this constraint, note that as $m \rightarrow 0, \alpha_{m} \sim 1$ and $\Gamma(m) \sim 1 / m$ so that $B(m / \chi, 5 / 2) \sim \chi / m$ and $\beta_{k} \sim 1 /(k+1)$. Using these facts in Eq. (27) gives

$$
P=\frac{\left(1-\phi_{0}\right)}{3 \pi^{2} B_{w}} \epsilon^{3 / 2}\left[n_{0}+\left(n_{\infty}-n_{0}\right)\left(1-\sum_{k=1}^{\infty} \frac{(-1)^{(k+1)}}{(k+1) !}\right)\right]
$$


But now, we also have

$$
e^{-1}=\sum_{k=0}^{\infty} \frac{(-1)^{k}}{k !}=\sum_{k=1}^{\infty} \frac{(-1)^{k+1}}{(k+1) !}
$$

since the first two terms of the expansion for $e^{-1}$ are 1 and -1 , which obviously cancel each other, so that

$$
P=\frac{\left(1-\phi_{0}\right)\left[n_{\infty}\left(1-e^{-1}\right)+n_{0} e^{-1}\right]}{3 \pi^{2} B_{w}} \epsilon^{3 / 2} .
$$

This is the same as Eq. (1) from Walton's theory [11] if $n_{w}=n_{\infty}\left(1-e^{-1}\right)+n_{0} e^{-1}$ is specified as the coordination number. Note that the coordination number given by Eq. (11) when $m \rightarrow 0$ is also $n_{\infty}\left(1-e^{-1}\right)+n_{0} e^{-1}$, which demonstrates internal consistency of the expressions. The bulk modulus as $m \rightarrow 0$ is thus given by the usual Walton [11] result of Eq. (3) with $n_{w}=n_{\infty}\left(1-e^{-1}\right)+n_{0} e^{-1}$.

In the limit that the strain $\epsilon \rightarrow 0$, Eq. (23) for the strain integral becomes

$$
J_{\epsilon} \sim B\left(\frac{m}{\chi}, \frac{5}{2}\right) \epsilon^{3 / 2+m / \chi} .
$$

From this fact, we obtain the low-strain limit of the functions $P(\epsilon)$ and $K(\epsilon)$

$$
\begin{aligned}
& P \sim \frac{\left(1-\phi_{0}\right) n_{\infty} \epsilon^{3 / 2}}{3 \pi^{2} B_{w}}\left[\left(1-\frac{n_{0}}{n_{\infty}}\right) \frac{3 \kappa_{m}}{3+2 m / \chi}\left(\frac{\epsilon}{\epsilon_{t}}\right)^{m / \chi}+\frac{n_{0}}{n_{\infty}}\right], \\
& K \sim \frac{\left(1-\phi_{0}\right) n_{\infty} \epsilon^{1 / 2}}{6 \pi^{2} B_{w}}\left[\left(1-\frac{n_{0}}{n_{\infty}}\right) \kappa_{m}\left(\frac{\epsilon}{\epsilon_{t}}\right)^{m / \chi}+\frac{n_{0}}{n_{\infty}}\right],
\end{aligned}
$$

where we have used the definition of the transition strain $\epsilon_{t}=\alpha_{m}^{-m / \chi}$ as given in Eq. (13). For close packs, one has $n_{0} / n_{\infty} \rightarrow 1$ and a classic Hertzian dependence $K \propto \epsilon^{1 / 2}$ emerges at low strain, which corresponds to a pressure dependence of $K \propto P^{1 / 3}$. This dependence was observed in the low-strain experiments of Jia and Mills [16], who carefully compacted their random bead packs so that $n_{0} / n_{\infty} \rightarrow 1$ prior to their measurement of wave speeds. For random loose packs (or random very loose packs), we have $n_{0} / n_{\infty} \rightarrow 0$ at zero stress, which results in the non-Hertzian strain dependence $K \propto \epsilon^{(1+2 m / \chi) / 2}$, corresponding to a pressure dependence of $K \propto P^{(1+2 m / \chi) /(3+2 m / \chi)}$. As presented earlier, several experimental investigations on random loose packs have found low-strain bulk moduli increasing faster than the Hertzian dependence and Eq. (36) may help to explain these observations. In particular, note that when $\chi=2$ (rotation) and $m=1$ in a loose pack or when $\chi=1$ (linear strain accumulation) and $m=1 / 2$, we recover the sometimes observed dependence of $K \propto \epsilon$, which is equivalent to $K \propto P^{1 / 2}$.

In the opposite limit where the strain $\epsilon$ becomes large, Eq. (23) for the strain integral becomes

$$
J_{\epsilon} \sim \epsilon^{3 / 2} \int_{0}^{\epsilon} s^{m / \chi-1} \mathrm{e}^{-\alpha_{m} s^{m / \chi}} \mathrm{d} s=\frac{\chi \epsilon^{3 / 2}}{m \alpha_{m}} \int_{0}^{\alpha_{m} \epsilon^{m / \chi}} \mathrm{e}^{-u} \mathrm{~d} u \sim \frac{\chi \epsilon^{3 / 2}}{m \alpha_{m}}
$$

From Eq. (22), we then obtain, for large $\epsilon$, the results:

$$
\begin{gathered}
P \sim \frac{\left(1-\phi_{0}\right) n_{\infty}}{3 \pi^{2} B_{w}} \epsilon^{3 / 2}, \\
K \sim \frac{\left(1-\phi_{0}\right) n_{\infty}}{6 \pi^{2} B_{w}} \epsilon^{1 / 2},
\end{gathered}
$$

which are just Walton's [11] formulas in Eqs. (1) and (3) with $n_{w}=n_{\infty}$. At large enough strain, all grains are jammed and the pressure dependence is ultimately Hertzian, as expected.

With these limits hereby established analytically, we use Eqs. (29) and (27) in Fig. 2 to plot the bulk modulus of a random loose pack $\left(n_{0} / n_{\infty}=0\right)$, as a function of pressure. Also displayed in the figure are the data of Murphy [2], as well as the Walton prediction [11]. The best fit to the data occurred using parameter values of $\chi=1$ (for the linear strain mechanism of gap closure), $m=1$ (a flat distribution for the gaps), and ratio 


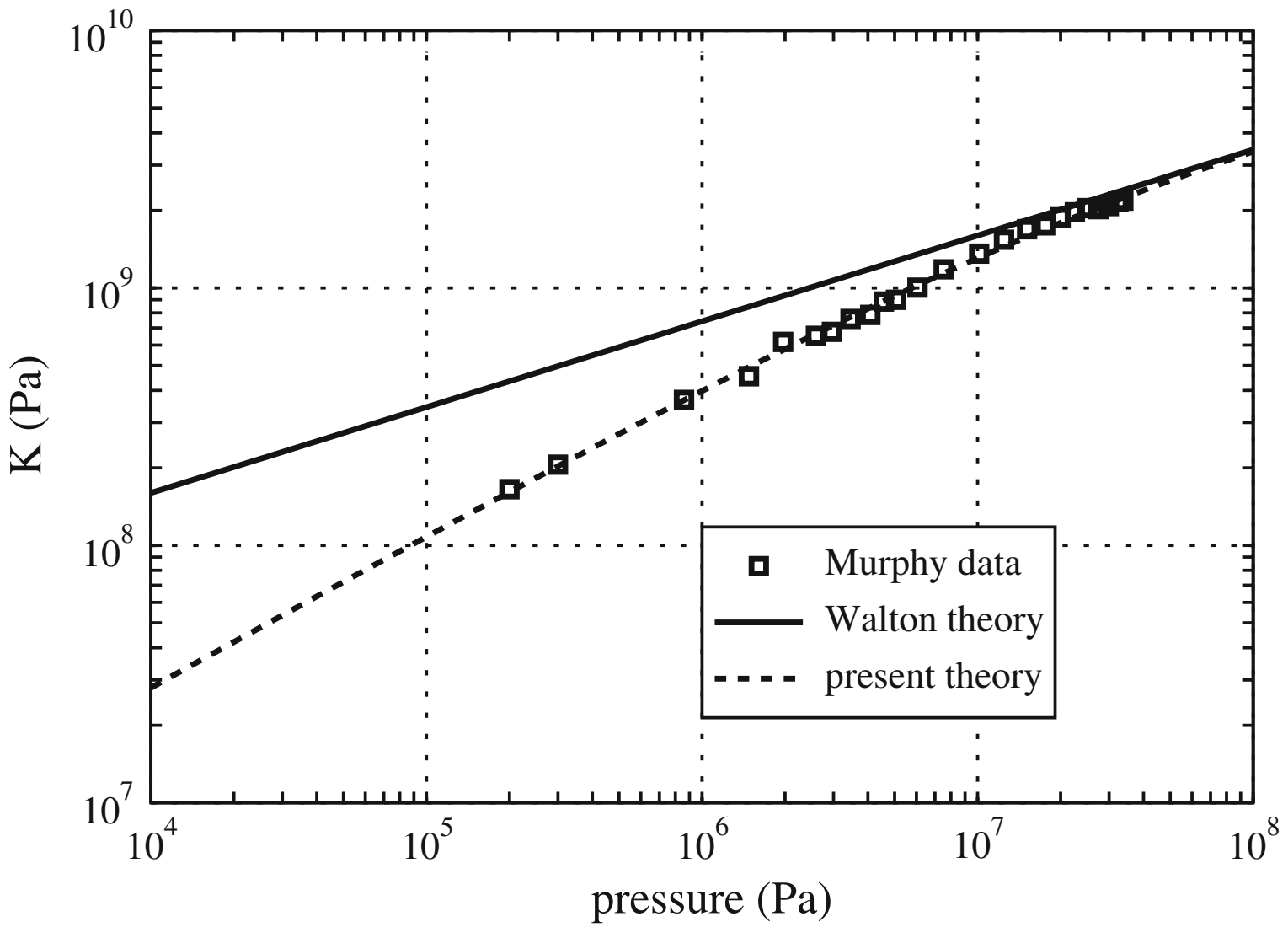

Fig. 2 The bulk modulus of a grain pack as a function of pressure. The dashed line was obtained using Eqs. (29) and (27) with model parameters $\chi=1, m=1$ and $D / h=300$, while the solid line is the result of Walton [11] and given by Eq. (3). These choices correspond to $K(P) \propto P^{3 / 5}$ at low pressures (note that $\frac{1}{3}<\frac{1}{2}<\frac{3}{5}$ ). Also see Eqs. (40)-(42) and subsequent discussion. The other parameters in the model were taken from Murphy [2] to be: $K_{s}=40.7 \mathrm{GPa}, G_{s}=29.7 \mathrm{GPa}, \phi_{0}=0.39, n_{0}=0$, and $n_{\infty}=12$

$D / h=300$. These choices correspond to a pressure dependence of $K \propto P^{3 / 5}$ at low pressures. The grain diameters in the Murphy [2] experiments were roughly $D=300 \mu \mathrm{m}$, which means that the maximum gap size between rattlers and their neighbors is on the order of $h=1 \mu \mathrm{m}$, which—although small—does not seem unreasonable. Note that if we had used $\chi=2$ (for the rotation mechanism), we would obtain the identical fit to the data so long as $m=2$ and, from Eq. (12), $D / h=\sqrt{150} \approx 12.25$, corresponding to $h \simeq 24.5 \mu \mathrm{m}$. The difficulty with using $m=2$ in Eq. (8) is that this choice corresponds to larger gaps being more probable than smaller gaps. This does not seem physically sensible for real grain packs, and therefore leads to the conclusion that the mechanism of gap closure through simple linear-strain accumulation is more likely to occur in the grain packs than is the grain-rotation mechanism. More discussion on this point will be provided with the conclusions.

Finally, we also obtain an approximate version of Eqs. (27) and (29) that gives both $P(\epsilon)$ and $K(\epsilon)$ without needing to evaluate either series. This result is obtained by connecting the known low- and high-strain limits found above with simple smooth (phenomenological) functions of the form:

$$
\begin{gathered}
P(\epsilon) \approx \frac{\left(1-\phi_{0}\right) n_{\infty} \epsilon^{3 / 2}}{3 \pi^{2} B_{w}}\left[\frac{n_{0} / n_{\infty}}{\left\{1+\left(\epsilon / \epsilon_{t}\right)^{a}\right\}^{1 / a}}+\frac{v_{P}\left(\epsilon / \epsilon_{t}\right)^{m / x}}{\left\{1+\left[v_{P}\left(\epsilon / \epsilon_{t}\right)^{m / \chi}\right]^{a}\right\}^{1 / a}}\right], \\
K(\epsilon) \approx \frac{\left(1-\phi_{0}\right) n_{\infty} \epsilon^{1 / 2}}{6 \pi^{2} B_{w}}\left[\frac{n_{0} / n_{\infty}}{\left\{1+\left(\epsilon / \epsilon_{t}\right)^{a}\right\}^{1 / a}}+\frac{v_{K}\left(\epsilon / \epsilon_{t}\right)^{m / x}}{\left\{1+\left[v_{K}\left(\epsilon / \epsilon_{t}\right)^{m / x}\right]^{a}\right\}^{1 / a}}\right],
\end{gathered}
$$


where

$$
v_{K}=\left(1-n_{0} / n_{\infty}\right) \kappa_{m} \quad \text { and } \quad v_{P}=\frac{3 v_{K}}{3+2 m / \chi} .
$$

The parameter $a$ controls the smoothness of the transition from the low-strain to high-strain limits. Smaller $a$ values correspond to gradual transitions and larger values to more abrupt transitions. Empirically, we find that using the coefficient $a=1.5$ produces a graphical fit to the data in Fig. 2 that is imperceptibly different from the complete expressions given by Eqs. (27) and (29).

In the loose-pack limit where $n_{0} / n_{\infty} \rightarrow 0$, Eqs. (35) and (36) can be combined to give a single formula for $K(P)$ variation at low pressures, while the Walton [11] result holds for $K(P)$ (with $n_{w}=n_{\infty}$ ) at high pressures. Again, combining these two limits with a simple function of pressure gives

$$
K(P)=\frac{\left(1-\phi_{0}\right) n_{\infty} \kappa_{m} \alpha_{m}^{-\chi /(2 m)}}{6 \pi^{2} B_{w}}\left(\frac{P}{P_{t}}\right)^{1 / 3} \frac{\left(P / P_{t}\right)^{\gamma}}{\left\{1+\left[\Omega\left(P / P_{t}\right)^{\gamma}\right]^{b}\right\}^{1 / b}},
$$

where the transition pressure $P_{t}$ is given by

$$
P_{t}=\frac{\left(1-\phi_{0}\right) n_{\infty}}{3 \pi^{2} B_{w}} \frac{3 \kappa_{m}}{3+2 m / \chi} \alpha_{m}^{-3 \chi /(2 m)},
$$

the exponent $\gamma$ is defined by

$$
\gamma=\frac{4 m / \chi}{3(3+2 m / \chi)}
$$

and the multiplier $\Omega$ is defined by

$$
\Omega=\left[\frac{\kappa_{m}^{2}(3+2 m / \chi)}{3}\right]^{1 / 3} .
$$

Note that $\frac{1}{3}+\gamma=\frac{1+2 m / \chi}{3+2 m / \chi}$, and then compare to the ratios of the exponents in Eqs. (35) and (36). A transition exponent of $b=2.5$ was empirically determined to give a fit to Murphy's data [2] in Fig. 2 that is equivalent to Eqs. (27) and (29). Equation (43) generalizes and improves upon a result given by Pride [4], which was based on grain-rotation alone having an exponent of $m=1$ in the probability distribution of gap sizes.

\section{Conclusions}

Analytical expressions were obtained for average pressure and bulk modulus variations with isotropic strain in random packings of grains. One key feature of the model is to allow the gaps between rattlers and jammed grains to close with increasing strain, thus creating new force-bearing contacts that act to stiffen the pack. The main approximation used to arrive at the analytical results is that strain is everywhere uniform throughout the pack. The situation in real grain packs differs from this since local strain is non-uniform with least strain present in regions that become jammed early in the strain history and greatest strain (when measured from the initial state) present in regions that jam later in the consolidation process. Maximum local strain in the pack will necessarily occur in the vicinities of the remaining rattler grains. Incorporating more realistic non-uniform strain distribution into the formalism presented here is possible, and will become a subject of future study.

A consequence of the uniform-strain approximation is that the greatest stress will exist in those regions that become jammed earliest in strain history. Because the average coordination number throughout the pack is known to increase most rapidly early in strain history, the present model based on uniform strain predicts that more contacts will have larger stress than lower stress. Such predictions differ from what is experimentally observed in real grain packs $[6,7,24]$ with most studies reporting an exponential fall-off in the number of contacts as a function of the force they carry. Accurate modeling of the grain-to-grain force partitioning will ultimately require incorporation of non-uniform strain distributions into a modified version of the modeling approach presented here.

With this caveat in mind, we have shown nevertheless that creation of new force-bearing contacts through closure of gaps between grains provides a very plausible explanation of the pressure dependence of measured 
bulk modulus in grain packs being different from the Hertzian prediction. Furthermore, by allowing explicitly for the randomness in the pack with a probability distribution for the gap sizes to be closed, we can explain results of various experimental studies that have measured different power-law pressure dependencies at low strain levels. We also propose physical mechanisms for why these different dependencies should be present, and why they produce different power-law behaviors.

Our theory has been successful in fitting the experimental data of Murphy [2] using reasonable values of three fitting parameters: (1) the power-law exponent $m$ for the probability of gap sizes [as given by Eq. (8)], (2) the index $\chi$ having value 1 if the mechanism of gap closure is linear strain accumulation or the value 2 if the mechanism is grain rotation, and (3) the ratio of grain diameter to maximum gap size $D / h$. For the gap-size distribution either to be flat $(m=1)$ or to have more small gaps than large gaps $(m<1)$, we found it necessary to choose $\chi=1$ (corresponding to the strain-accumulation mechanism of gap closure) in order to fit the data. For the present theory to fit any data for which the moduli increase faster than $P^{1 / 2}$ (such as the data of Murphy [2] and Kuwano and Jardine [15]) while requiring that $m \leq 1$, we must necessarily use the strain-accumulation mechanism $(\chi=1)$ and not the grain-rotation mechanism $(\chi=2)$. However, until non-uniform strain is successfully incorporated into the present formalism, some doubt remains as to the relative importance of the various force-chain building mechanisms, especially in light of the great variety of grain-packing scenarios that are available. The formalism outlined here permits analytical treatment of several of the most pertinent scenarios.

The present model is based on Hertzian contact mechanics and is formulated to treat packings of identical spheres under isotropic applied strain. An actual sand pack within the earth may require further physical considerations not yet discussed. A non-uniform grain-size distribution would require modifying the local displacements of grain centers in Walton's theory [11]. This modification should be possible, along with allowing for each grain to have its own distinct elastic modulus, so long as such fluctuations are random and given by known distribution functions. The Hertzian contact mechanics acting at each contact requires that the two surfaces be quadratics prior to the growth of the contact area. This assumption breaks down when one or both of the grains have edges that are angular, in which case non-Hertzian contact mechanics based on, for example, wedge geometries must be employed (e.g., [25]). At large enough strain levels, plastic yielding and fracture of the grains must be considered. For grains made of common minerals like quartz and soda-lime glass that have moduli on the order of a few tens of GPa, fracture begins to occur at pressures of roughly 30-50 MPa. Fortunately, the important applications cited in the introduction occur at much smaller pressures. Generalizing to non-isotropic applied strain is relatively straightforward and was allowed for by Walton [11]. We did not consider anisotropic strain states here in order to keep the expressions and analysis algebraically simple. No mechanism for (likely) hysteretic effects of strain cycling was included. Some of the experimentally observed hysteresis is due to plastic deformation of small asperities on the grain surfaces and some is due to irreversible changes (e.g., sliding) in the packing arrangement. Finally, how porosity decreases as strain increases was not explicitly treated. This mechanism is fairly straightforward to model and will be included in future work.

Acknowledgments Work of both SRP and JGB was performed under the auspices of the U.S. Department of Energy by the University of California, Lawrence Berkeley National Laboratory under Contract No. DE-AC02-05CH11231 and supported specifically by the Geosciences Research Program of the DOE Office of Basic Energy Sciences, Division of Chemical Sciences, Geosciences and Biosciences.

Open Access This article is distributed under the terms of the Creative Commons Attribution Noncommercial License which permits any noncommercial use, distribution, and reproduction in any medium, provided the original author(s) and source are credited.

\section{References}

1. Hardin, B.O., Richart, F.E.: Elastic wave velocities in granular soils. J. Soil Mech. Found. Div. ASCE 89, 33-65 (1963)

2. Murphy III, W.F.: Effects of microstructure and pore fluids on the acoustic properties of granular sedimentary materials. Ph.D. thesis, Stanford University, Stanford, California (1982)

3. Berryman, J.G.: Effective stress for transport properties of inhomogeneous porous rock. J. Geophys. Res. 97, 17,40917,424 (1992)

4. Pride, S.R.: Relationships between seismic and hydrological properties. In: Rubin, Y., Hubbard, S. (eds.) Hydrogeophysics, pp. 253-291. Springer, The Netherlands (2005)

5. Drescher, A., de Josselin de Jong, G.: Photoelastic verification of a mechanical model for the flow of a granular material. J. Mech. Phys. Solids 20, 337-351 (1972)

6. Mueth, D.M., Jaeger, H.M., Nagel, S.R.: Force distribution in a granular medium. Phys. Rev. E 57, 3164 (1998) 
7. Erikson, J.M., Mueggenburg, N.W., Jaeger, H.M., Nagel, S.R.: Force distributions in three-dimensional compressible granular packs. Phys. Rev. E 66, 040,301(R) (2002)

8. Majmudar, T.S., Behringer, R.P.: Contact force measurements and stress-induced anisotropy in granular materials. Nature 435, 1079-1082 (2005)

9. Agnolin, I., Roux, J.: Internal states of model isotropic granular packings. II. Compression and pressure cycles. Phys. Rev. E 76, 061303-1-061303-11 (2007)

10. Goddard, J.D.: Nonlinear elasticity and pressure-dependent wave speeds in granular media. Proc. R. Soc. Lond. A 430, 105-131 (1990)

11. Walton, K.: The effective elastic moduli of a random packing of spheres. J. Mech. Phys. Solids 35, 213-226 (1987)

12. Landau, L.D., Lifshitz, E.M.: Theory of Elasticity. Butterworth-Heinemann, Oxford (1986)

13. Domenico, S.N.: Elastic properties of unconsolidated porous reservoirs. Geophysics 42, 1339-1368 (1977)

14. Makse, H.A., Gland, N., Johnson, D.L., Schwartz, L.: Granular packings: Nonlinear elasticity, sound propagation, and collective relaxation dynamics. Phys. Rev. E 70, 061302-1-061302-19 (2004)

15. Kuwano, R., Jardine, R.: On the applicability of cross-anisotropic elasticity to granular materials at very small strain. Geotechnique 52, 727-749 (2002)

16. Jia, X., Mills, P.: Sound propagation in dense granular materials. In: Kishino, Y. (ed.) Powders and Grain 2001, pp. $105-112$. Balkema, Rotterdam (2001)

17. Zimmer, M.A., Prasad, M., Mavko, G., Nur, A.: Seismic velocities of unconsolidated sands. Part 1. Pressure trends from 0.1 to $20 \mathrm{MPa}$. Geophysics 72, E1-E13 (2007)

18. Goddard, J.D.: On entropy estimates of contact forces in static granular assemblies. Int. J. Solids Struct. 41, 5851-5861 (2004)

19. Jiang, Y.M., Liu, M.: Energetic instability unjams sand and suspension. Phys. Rev. Lett. 93, 148001-1-148001-4 (2004)

20. Agnolin, I., Roux, J.: Internal states of model isotropic granular packings. III. Elastic properties. Phys. Rev. E 76, 061304-1-061304-22 (2007)

21. Agnolin, I., Roux, J.: On the elastic moduli of three-dimensional assemblies of spheres: Characterization and modeling of fluctuations in the particle displacement and rotation. Int. J. Solids Struct. 45, 1101-1123 (2008)

22. Gradshteyn, I.S., Ryzhik, I.M. (eds.): Tables of Integrals, Series, and Products. Academic Press, New York (1965)

23. Rudin, W.: Principles of Mathematical Analysis. McGraw-Hill Book Company, New York (1964)

24. Makse, H.A., Johnson, D.L., Schwartz, L.: Packing of compressible granular materials. Phys. Rev. Lett. 84, 4160-4163 (2000)

25. Johnson, K.L.: Contact Mechanics. Cambridge University Press, Cambridge (1985) 